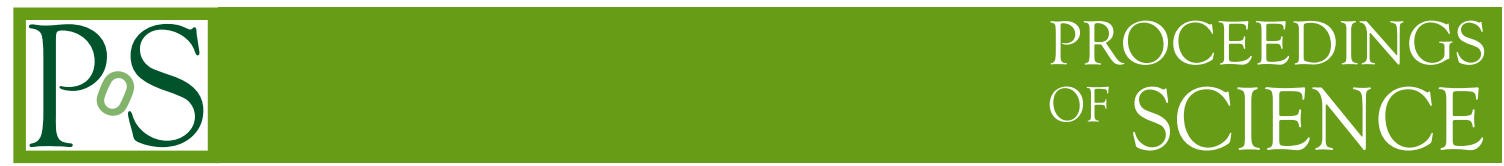

\title{
On the $N_{\mathrm{f}}$-dependence of gluonic observables
}

\section{Mattia Bruno* and Rainer Sommer}

NIC @ DESY. Platanenallee 6, 15738 Zeuthen, Germany

E-mail: mattia.bruno@desy.de

\section{for the ALPHA collaboration}

We compute $t_{0}, w_{0}$ and the topological susceptibility, defined at finite gradient flow time for two-flavour QCD. The use of three lattice spacings and pion masses between 192 and $500 \mathrm{MeV}$ together with a careful error analysis allow to approach the continuum limit of the two-flavour theory despite significant auto-correlations. A comparison to $N_{\mathrm{f}}=0$ results shows the size of sea quark effects in $t_{0}^{2} \chi$, with $\chi$ the topological susceptibility, and low energy observables such as $t_{0} / w_{0}^{2}$ and $t_{0} / r_{0}^{2}$.

Mainz, Germany

\footnotetext{
*Speaker.
} 


\section{Introduction}

In these Proceedings we investigate the effects of dynamical quarks on gluonic observables. In the high-energy sector the dominant effect of dynamical quarks is well understood in terms of the running of the coupling. To probe the low-energy sector we use the recently introduced gradient flow, which allows to define new renormalisation-group-invariant observables [1]. Here we concentrate on $t_{0}$ and $w_{0}$ as well as the topological susceptibility. We compute them with $N_{\mathrm{f}}=2$ light dynamical quarks using the CLS ensembles, based on $\mathrm{O}(a)$-improved Wilson fermions (see [2] for the set of ensembles), and we compare with $N_{\mathrm{f}}=0$ and $N_{\mathrm{f}}>2$ results from the literature. Clearly, the topological susceptibility is of particular interest since chiral perturbation theory predicts a suppression proportional to the quark mass for light sea quarks. However, critical slowing down of the topological modes makes the susceptibility particularly difficult to study [3]. Taken together this means that the susceptibility is an important indicator for the correctness of the simulations.

The gradient flow is defined by the following equation [1]:

$$
\begin{aligned}
\partial_{t} B_{\mu}(x, t) & =D_{v} G_{v \mu}(x, t), \quad B_{\mu}(x, 0)=A_{\mu}(x), \\
G_{\mu v} & =\partial_{\mu} B_{v}-\partial_{v} B_{\mu}+\left[B_{\mu}, B_{v}\right], \quad D_{\mu}=\partial_{\mu}+\left[B_{\mu}, \cdot\right],
\end{aligned}
$$

where $B_{\mu}(x, t)$ is the gauge field at positive flow time $t$ (which has dimension length squared). The energy density $\langle E(t)\rangle=-\frac{1}{2}\left\langle\operatorname{tr}\left\{G_{\mu \nu} G_{\mu \nu}\right\}\right\rangle$ has been used to define a scale $t_{0}$ via $\left.t^{2}\langle E(t)\rangle\right|_{t=t_{0}}=0.3$. In general, the correlation functions of the smooth field $B_{\mu}(x, t)$ do not need renormalisation at any separation in space-time. Therefore $q(x, t)=-\frac{1}{32 \pi^{2}} \varepsilon^{\mu v \rho \sigma} \operatorname{tr}\left\{G_{\mu v}(x, t) G_{\rho \sigma}(x, t)\right\}$ can be used directly to define the topological charge $Q(t)=a^{4} \sum_{x} q(x, t)$. We evaluate it at $t=t_{0}$ using the clover-type ("symmetric", cf. [1]) discretisation of $G_{\mu v}(x, t)$.

\section{Topology and auto-correlations}

Following [1] we check how strong the separation of topological sectors is realised with our lattice action. In principle the suppression of regions in configuration space between the charge sectors could be stronger or weaker than in the pure gauge theory. However, as for the pure Wilson gauge theory [1], we find that the probability of fields "between the sectors" goes to zero as $R_{0}(m)^{-10}$ also for our theory including dynamical Wilson fermions. By $R_{0}(m)$ we denote $r_{0} / a$ as a function of the quark mass, a measure for the gluonic correlation length of the system. Fig. 1, left, shows the scaling with $a$ at fixed pion mass, but we also verified the $R_{0}(m)^{-10}$ scaling as a function of the quark mass at fixed bare coupling.

The strong depletion of the configuration space between the sectors means that eventually the topological charge will not be properly sampled at all. In our case, the algorithm has difficulties and auto-correlations are large in particular for our $a=0.048 \mathrm{fm}$ ensembles. They have to be controlled in order to obtain reliable MC results and errors. We first look at the auto-correlation functions of the observables under study and, in order to quote a safe error estimate for our results, we follow the method developed in [3] to evaluate the auto-correlation times $\tau_{\exp }$ and $\tau_{\text {int }}$.

In Fig. 2 we plot some examples, for two lattice spacings, of the auto-correlation functions $\rho\left(t_{\mathrm{MC}}\right)$, normalised as $\rho(0)=1$, of $t_{0}$ and $Q^{2}\left(t_{0}\right)$. In both cases auto-correlations are under reason- 

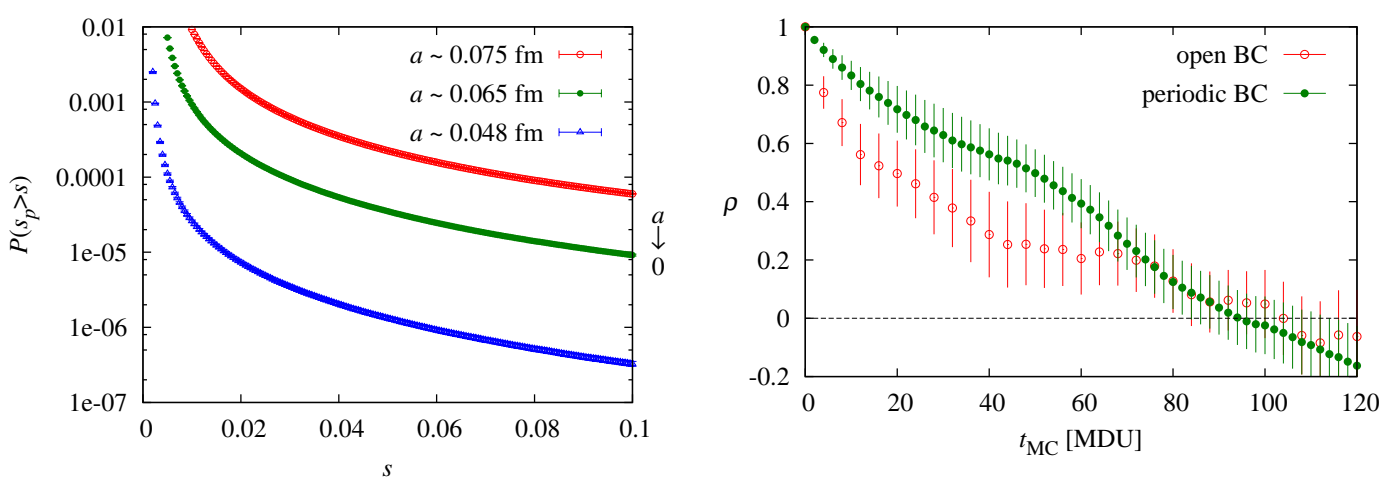

Figure 1: Left: probability of $s_{p}(t)=\operatorname{Re} \operatorname{tr}\left(1-V_{t}(p)\right)$ (where $V_{t}$ is the plaquette loop) to be bigger than a certain value $s$ at fixed $r_{0} m_{\pi} \approx 0.6$. Note that for $s_{p}<0.067$ the space of lattice fields consists of disconnected sectors [4]. Right: normalised auto-correlation function of $t_{0}$ with periodic and open BC with $a=0.075 \mathrm{fm}$, $m_{\pi} \approx 280 \mathrm{MeV}$ and roughly $1000 \mathrm{MDU}$.

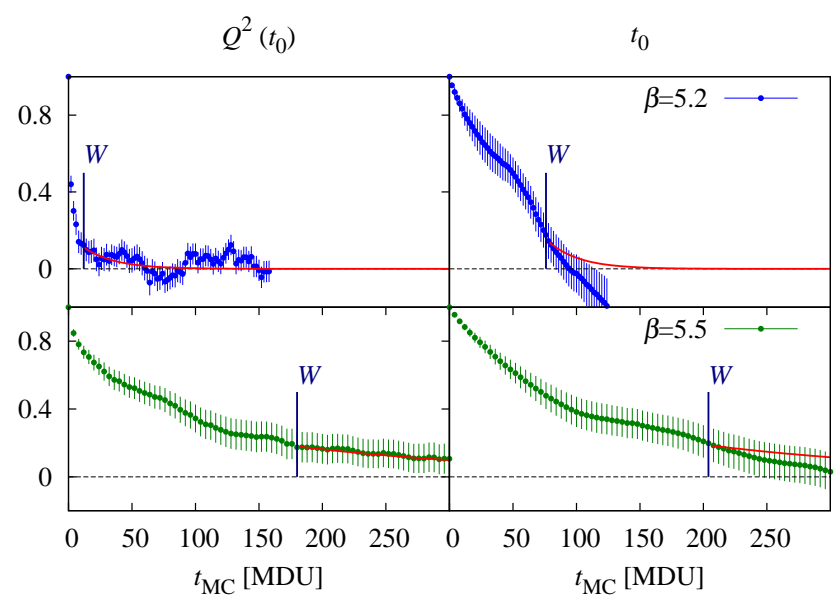

Figure 2: Normalised auto-correlation functions at $a=0.075 \mathrm{fm}, m_{\pi} \approx 280 \mathrm{MeV}$ (top), and at $a=0.048 \mathrm{fm}$, $m_{\pi}=340 \mathrm{MeV}$ (bottom). The red curves are our estimates of the contribution of the tails of $\rho$.

able control. For our lattice spacings, $t_{0}$ shows larger auto-correlations than the topological charge. It is in fact a good estimator of the exponential auto-correlation time, better than the $Q^{2}$ used in [3].

Recent studies [5] showed that when open boundary conditions (along the Euclidean time) are employed, the MC sampling of topology is significantly accelerated. We observe that, at our largest lattice spacing, the ALPHA Collaboration open BC simulation has similar auto-correlations as the periodic BC one, as shown in Fig. 1, right. Note that the two simulations do not use exactly the same algorithm.

A further very useful test of the quality of the ensembles is to look at the distributions of the topological charge. We define the observable

$$
f_{v}(Q)=\theta\left(Q-\left(v-\frac{1}{2}\right)\right) \theta\left(v+\frac{1}{2}-Q\right), \quad v \in \mathbb{N},
$$

whose mean value $\left\langle f_{v}(Q)\right\rangle=P(v)$ is the probability of the topological charge to be in the unit 

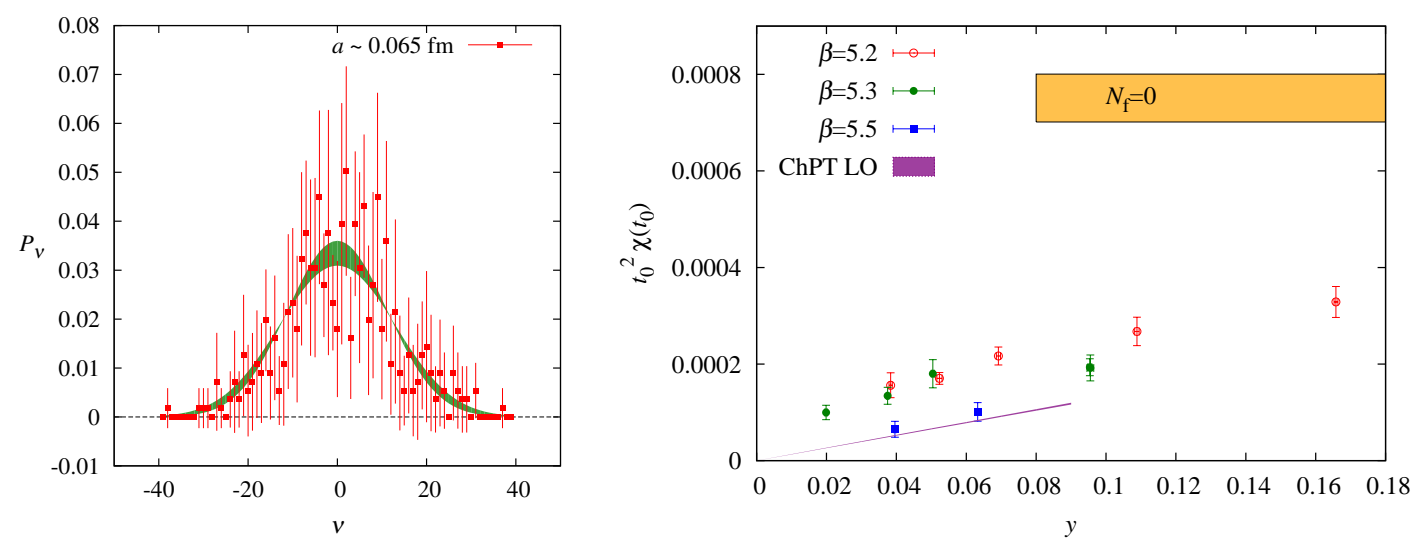

Figure 3: Left: probability distribution of the topological charge at $t=t_{0}$ of our intermediate lattice spacing. For this ensemble $m_{\pi}=192 \mathrm{MeV}$ and $t_{\mathrm{MC}}=1100 \mathrm{MDU}$. Right: the susceptibility in units of $t_{0}$; the large mass asymptotic value from $N_{\mathrm{f}}=0$ is indicated as a horizontal error band, while the lowest order chiral perturbation theory prediction (using our results for $t_{0} f_{\pi}^{2}$ ) is the purple band.

length interval around $v$. It is a standard observable whose error can be computed as above. In large volume $P(v)$ approaches a Gaussian [6]. We find this well realised when the Monte Carlo history is at least $\approx 20 \tau_{\text {exp }}$, (e.g. Fig. 3, left). Our errors render corrections to the gaussian behaviour invisible.

We now restrict ourselves to the ensembles with a length of at least $\approx 20 \tau_{\exp }$ and investigate the quark mass dependence of the susceptibility $\chi\left(t_{0}\right)=\left\langle Q^{2}\left(t_{0}\right)\right\rangle /\left(L^{3} \cdot T\right)$. From the comparison to the $N_{\mathrm{f}}=0$ result $[7,1]$ in Fig. 3, right, the strong suppression of the susceptibility caused by the sea quarks is evident. However, also lattice spacing effects are clearly visible. Note that $\chi$ has dimension (mass) ${ }^{-4}$, where scaling violations, e.g. simply induced through the scale setting, are strongly enhanced. Similar scaling violations have been observed in [8]. When the lattice spacing is reduced down to $a=0.048 \mathrm{fm}$, we obtain a result in rough agreement with the leading order of the chiral expansion of the susceptibility

$$
\chi=\frac{m}{2} \Sigma(1+\mathrm{O}(m))=\frac{f_{\pi}^{2} m_{\pi}^{2}}{8}+\mathrm{O}\left(m_{\pi}^{4}\right) .
$$

\section{Scales from the gradient flow}

In scale setting [9], statistically precise scales that mildly depend on the quark masses are particularly welcome. A scale closely related to $t_{0}$ is $w_{0}$, defined by [10] $\left.t \frac{d}{d t}\left[t^{2} E(t)\right]\right|_{t=w_{0}^{2}}=0.3$. Despite the large auto-correlations shown above, both $t_{0}$ and $w_{0}$ are more precise than $r_{0}$ [11]; their variance is very small.

At fixed $\beta$ a dependence on the renormalised quark mass is present but not very strong. We linearly extrapolate the three scales using the quantity $y=m_{\pi}^{2} t_{0}$, defined at finite mass, as shown in Fig. 4, left. In the future we will incorporate the asymptotic behaviour of chiral perturbation theory into the extrapolation of $t_{0}$ [12]. An interesting question is whether there are mass-dependent cutoff effects. None of these are visible in ratios such as $t_{0} / t_{0, \text { ref }}$ (Fig. 4 , right). Within our good precision this ratio is described by a universal curve. At this point we note that in our $\mathrm{O}(a)$ improved action 

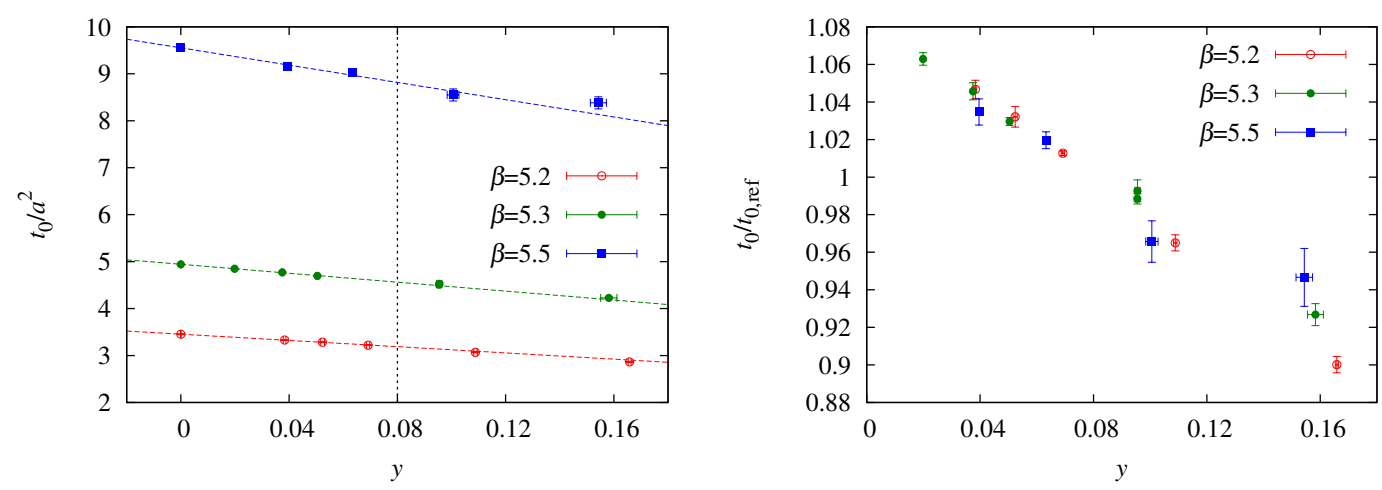

Figure 4: Left: behaviour of $t_{0} / a^{2}$ as a function of the pion mass; to have control on the extrapolation we use different functions and ranges in $y$ and we quote, as final result, the linear extrapolation with $y \leq 0.08$, corresponding to $m_{\pi} \leq 390 \mathrm{MeV}$. Right: for each $\beta$, $t_{0, \text { ref }}$ is the value of $t_{0}$ interpolated to $y=0.08$.

we have neglected a small term $a b_{\mathrm{g}} m_{\mathrm{q}} \operatorname{tr} F_{\mu \nu} F_{\mu \nu}$ [13]. The reason to neglect it was that both $b_{\mathrm{g}}$ is very small at 1-loop [14] and the bare subtracted quark masses $a m_{\mathrm{q}}$ are very small. We can now verify that, with few-per-mille precision, no residual $\mathrm{O}(a)$ effects are present in $t_{0}$. All statements made hold also for $w_{0}$ and $r_{0}$, apart from a worse precision for the latter.

To convert our results to physical units we use the lattice spacings of [15], based on $f_{\mathrm{K}}=155$ $\mathrm{MeV}$. We then extrapolate linearly in $a^{2} / t_{0}$ to the continuum limit, finding:

$$
\begin{array}{cl}
t_{0}^{\text {chiral }} & =0.02396(37) \mathrm{fm}^{2}, \quad w_{0}^{\text {chiral }}=0.1776(13) \mathrm{fm}, \\
t_{0}^{\text {phys }} & =0.02356(36) \mathrm{fm}^{2}, \quad w_{0}^{\text {phys }}=0.1757(13) \mathrm{fm},
\end{array}
$$

where "phys" indicates the physical point, given by the physical pion mass (and $f_{\mathrm{K}}$ ). A comparison to other determinations of these scales in physical units needs care, since it depends on how that scale was set originally, e.g. in [10] the mass of the $\Omega$ baryon was used. A proper discussion of the dependence on the number of flavours requires to consider specific ratios. We now turn to those.

\section{Dynamical quark effects}

The three possible ratios obtained by combining $t_{0} / a^{2},\left(r_{0} / a\right)^{2}$ and $\left(w_{0} / a\right)^{2}$ are extrapolated to the physical point as discussed above. We then approach the limit $a \rightarrow 0$ of all three ratios by a linear extrapolation in $a^{2} / t_{0}$, shown in Fig. 5. Here $t_{0} / r_{0}^{2}$ has the smallest discretisation effects. The Figure also shows a comparison with results with a different number of flavours. Those for $N_{\mathrm{f}}=0$ either come directly from [1] or from our analysis of $\langle E(t)\rangle$ computed there. For $N_{\mathrm{f}}=2+1$, we estimated $t_{0} / r_{0}^{2}$ and $w_{0}^{2} / r_{0}^{2}$ by combining $r_{0}=0.480(11) \mathrm{fm} \mathrm{[16]} \mathrm{with} \sqrt{t_{0}}=0.1465(25) \mathrm{fm}$ and $w_{0}=0.1755(18) \mathrm{fm}[10]$. Also $t_{0} / w_{0}^{2}$ is computed from those numbers neglecting error correlations, which most certainly yields a strong overestimate of the error. Finally for $N_{\mathrm{f}}=2+1+1$ we combine $r_{0} / r_{1}=1.508(5)$ [17] (neglecting a difference between $N_{\mathrm{f}}=2+1$ and $N_{\mathrm{f}}=2+1+1$ ), $\sqrt{t_{0}} / w_{0}=0.835(8)$ and $r_{1} / w_{0}=1.790(25)$ [18].

The ratios shown in Fig. 5 demonstrate that the $N_{\mathrm{f}}=0$ and the $N_{\mathrm{f}}=2$ theories differ quite strongly for these purely gluonic observables. This is interesting since we are looking at infrareddominated quantities - non-perturbative features of the theory. The effects of the heavier quarks, 


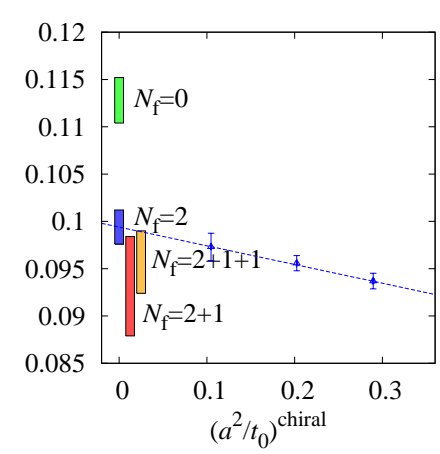

(a) $t_{0} / r_{0}^{2}$

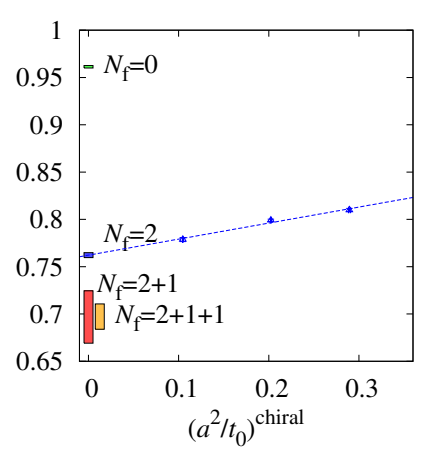

(b) $t_{0} / w_{0}^{2}$

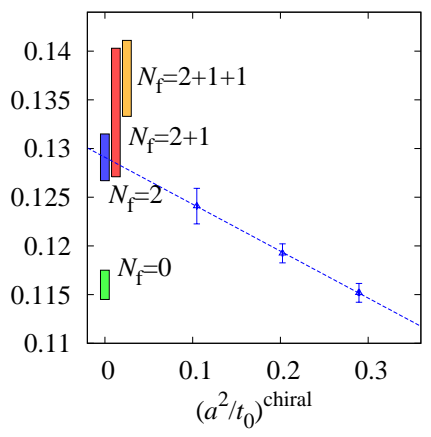

(c) $w_{0}^{2} / r_{0}^{2}$

Figure 5: Continuum extrapolation and flavour number dependence of ratios of scales.

strange and charm, appear to be less pronounced, but still noticeable. Of course, for a very heavy quark, decoupling is expected in the sense that such dimensionless low energy quantities should agree for theories with $N_{\mathrm{f}}$ and $N_{\mathrm{f}}-1$ quarks up to corrections suppressed by inverse powers of the mass $m_{N_{\mathrm{f}}}$ of the heaviest quark.

\section{Conclusions}

In this work we have studied the topological charge and the scales $t_{0}$ and $w_{0}$ for $N_{\mathrm{f}}=2 \mathrm{O}(a)$ improved Wilson fermions. We demonstrated the quality of our ensembles via empirical tests such as the distribution of the topological charge and its susceptibility. Both turn out to be in agreement with theoretical expectations, even if the latter is affected by large discretisation effects. We verified that when the statistics is at least $20 \tau_{\exp }$ auto-correlations are under reasonable control and error estimates are possible down to lattice spacings $\approx 0.05 \mathrm{fm}$. The dynamical separation of the topological sectors in the $N_{\mathrm{f}}=2$ theory is very similar to the pure gauge theory.

The expected suppression of topology by dynamical fermions is observed. We investigated the $N_{\mathrm{f}}$ dependence of $t_{0}, w_{0}$ and $r_{0}$ by a comparison to data in the literature. The use of the full CLS ensembles and the mild dependence on the quark mass allowed for a controlled extrapolation to the two-flavour continuum theory. Ratios of the scales show a rather significant effect of the two light dynamical fermions, but, interestingly, already the effect of the heavier strange quark is not as pronounced.

\section{Acknowledgments}

We would like to thank M. Lüscher for sharing the $N_{\mathrm{f}}=0$ data, M. Della Morte, T. Korzec and S. Schaefer for the configurations of the openBC ensemble. We had access to HPC resources in the form of a regular GCS/NIC project ${ }^{1}$, a JUROPA/NIC project $^{1}$ and through PRACE-2IP, receiving funding from the European Community's Seventh Framework Programme (FP7/2007-2013) under grant agreement RI-283493. This work is supported in part by the grants SFB/TR9 of the Deutsche Forschungsgemeinschaft.

\footnotetext{
${ }^{1}$ http://www.fz-juelich.de/ias/jsc/EN/Expertise/Supercomputers/ComputingTime/Acknowledgements.html
} 


\section{References}

[1] M. Lüscher, Properties and uses of the Wilson flow in lattice QCD, JHEP 1008 (2010) 071, [arXiv:1006.4518].

[2] S. Lottini, Chiral behaviour of the pion decay constant in $N_{f}=2 Q C D, P o S$ LATTICE2013 (2013) 315, [arXiv:1311.3081].

[3] ALPHA Collaboration Collaboration, S. Schaefer, R. Sommer, and F. Virotta, Critical slowing down and error analysis in lattice QCD simulations, Nucl.Phys. $\mathbf{B 8 4 5}$ (2011) 93-119,

[arXiv:1009.5228].

[4] M. Lüscher, Topology of Lattice Gauge Fields, Commun.Math.Phys. 85 (1982) 39.

[5] M. Lüscher and S. Schaefer, Lattice QCD without topology barriers, JHEP 1107 (2011) 036, [arXiv:1105.4749].

[6] L. Giusti, M. Lüscher, P. Weisz, and H. Wittig, Lattice QCD in the epsilon regime and random matrix theory, JHEP 0311 (2003) 023, [hep-lat/ 030918 9].

[7] L. Del Debbio, L. Giusti, and C. Pica, Topological susceptibility in the SU(3) gauge theory, Phys.Rev.Lett. 94 (2005) 032003, [hep-th/ 0407052 ].

[8] MILC collaboration Collaboration, A. Bazavov et al., Topological susceptibility with the asqtad action, Phys.Rev. D81 (2010) 114501, [arXiv:1003.5695].

[9] R. Sommer, Scale Setting in Lattice QCD, PoS LATTICE2013 (2013) 015, [arXiv: 1401.3270 ].

[10] S. Borsanyi, S. Durr, Z. Fodor, C. Hoelbling, S. D. Katz, et al., High-precision scale setting in lattice QCD, JHEP 1209 (2012) 010, [arXiv:1203.4469].

[11] R. Sommer, A new way to set the energy scale in lattice gauge theories and its applications to the static force and $\alpha_{s}$ in SU(2) Yang-Mills theory, Nucl. Phys. B411 (1994) 839-854, [hep-lat/9310022].

[12] M. Lüscher, Future applications of the Yang-Mills gradient flow in lattice QCD, PoS LATTICE2013 (2013) 016, [arXiv:1308.5598].

[13] M. Lüscher, S. Sint, R. Sommer, and P. Weisz, Chiral symmetry and $O($ a) improvement in lattice QCD, Nucl. Phys. B478 (1996) 365-400, [hep-lat/9605038].

[14] S. Sint and R. Sommer, The Running coupling from the QCD Schrodinger functional: A One loop analysis, Nucl.Phys. B465 (1996) 71-98, [hep-lat/9508012].

[15] P. Fritzsch, F. Knechtli, B. Leder, M. Marinkovic, S. Schaefer, et al., The strange quark mass and Lambda parameter of two flavor QCD, Nucl.Phys. B865 (2012) 397-429, [arXiv: 1205.5380 ].

[16] RBC Collaboration, UKQCD Collaboration Collaboration, R. Arthur et al., Domain Wall QCD with Near-Physical Pions, Phys.Rev. D87 (2013) 094514, [arXiv: 1208 . 4412].

[17] A. Bazavov, T. Bhattacharya, M. Cheng, C. DeTar, H. Ding, et al., The chiral and deconfinement aspects of the QCD transition, Phys.Rev. D85 (2012) 054503, [arXiv:1111.1710].

[18] R. Dowdall, C. Davies, G. Lepage, and C. McNeile, Vus from pi and K decay constants in full lattice QCD with physical $u, d$, s and c quarks, Phys.Rev. D88 (2013) 074504, [arXiv:1303.1670]. 